\title{
Browning of white fat: does irisin play a role in humans?
}

Manuela Elsen, Silja Raschke and Jürgen Eckel

Paul-Langerhans-Group for Integrative Physiology, German Diabetes Center, Auf'm Hennekamp 65, 40225 Düsseldorf, Germany
Correspondence should be addressed to J Eckel

Email eckel@hhu.de

\begin{abstract}
The discovery of irisin as an exercise-regulated myokine inducing browning of WAT has gained interest as a potential new strategy to combat obesity and its associated disorders, such as type 2 diabetes. However, there are inconsistencies regarding the relevance of irisin in humans. The regulation of FNDC5 mRNA expression by exercise and contraction could not be reproduced by a number of human studies using several exercise protocols and in vitro approaches. Furthermore, the nature of FNDC5 fragments and the presence of irisin in humans are questionable and probably contribute to conflicting data obtained with commercially available ELISA kits. Most importantly, the information regarding the concentration of circulating irisin in humans is not clear, as different studies using different kits measure irisin levels in a wide range. Data about the role of irisin in states of human obesity and metabolic diseases are conflicting and, in some cases, changes in irisin levels have been observed; they were only moderate in 10-20\%. Independent of the presence and regulation of FNDC5/irisin in humans, the application of recombinant irisin could still represent a therapeutic strategy to fight obesity. However, the current data obtained from human cell models reveal that FNDC5/irisin has no effect on browning of the major WAT depots in humans and is likely to selectively target a small subpopulation of adipocytes, which are located in classical BAT regions, such as the supraclavicular adipose tissue. Thus, other candidates, such as BMP7 or CNPs, seem to be more prominent candidates as inducers of browning in humans.
\end{abstract}
Key Words
- adipocyte
- diabetes
- exercise
obesity
- muscle

Journal of Endocrinology (2014) 222, R25-R38

\section{Introduction}

White adipose tissue (WAT) and brown adipose tissue (BAT) exert inverse functions in that WAT stores energy, whereas BAT, characterized by a large number of mitochondria containing uncoupling protein 1 (UCP1), mediates adaptive thermogenesis and contributes to the maintenance of body temperature. In 2009, five independent studies demonstrated the presence of BAT in adult humans (Cypess et al. 2009, van Marken Lichtenbelt et al. 2009, Saito et al. 2009, Virtanen et al. 2009, Zingaretti et al. 2009) and confirmed the existence of an inverse correlation between BMI and BAT activity. These observations have aroused considerable interest in the therapeutic potential of brown adipocytes for inducing weight loss, and recent data have confirmed that BAT oxidative metabolism contributes significantly to energy expenditure (Ouellet et al. 2012). The classical view of brown and white fat cells was modified in 2010 by a publication of the Cannon/Nedergaard group describing a third type of fat cells termed 'brite' (brown-in-white) adipocytes (Petrovic et al. 2010). These cells share biochemical features and the thermogenic potential with brown adipocytes, but are derived from different precursor cells (Seale et al. 2008).

Published by Bioscientifica Ltd. 
The possibility to differentiate adipose stem cells into brite adipocytes (browning of white fat) and to induce thermogenic activation and augment energy expenditure is currently considered as an important promising approach to combat obesity and obesity-related complications.

A novel hormone-like myokine termed irisin has recently been described to activate such a white-tobrown shift in adipocytes (Boström et al. 2012a). As this molecule was originally reported to be released after physical activity, it gained huge interest as a potential mediator of the health-promoting effects of physical exercise. Irisin is a 112 amino acid peptide cleaved from fibronectin type III domain containing protein 5 (FNDC5), a type I membrane protein which was claimed to be upregulated by exercise training in both mice and humans (Boström et al. 2012a). When writing this review, more than 100 papers dealing with irisin have been found in PubMed. However, the physiological role and the potential therapeutic value of irisin have remained highly controversial. In this review, we analyzed the current literature regarding exercise regulation and the functional impact of irisin in humans. We conclude that: i) the nature and concentration of circulating FNDC5 fragments remain unclear and ii) irisin has no effect on major WAT depots in humans and may only target a small subpopulation of adipocytes.

\section{Impact of exercise on browning of white adipose tissue}

As noticed before, the exercise-regulated myokine irisin was identified and described as a link between exercise and the promotion of WAT browning (Boström et al. 2012a). It is well known that the transcriptional coregulator PGC1 $\alpha$ is induced in muscle in response to exercise in rodents and humans (Goto et al. 2000, Pilegaard et al. 2003). Therefore, transgenic mice overexpressing Pgc1a (Ppargc1a) in skeletal muscle can be used as an exercise model (Boström et al. 2012a). Interestingly, these transgenics exhibited increased expression of the brown marker genes $U c p 1$ and cell death-inducing DFFA-like effector A (Cidea) only in the inguinal depot, suggesting that muscle activity promotes remodeling of subcutaneous WAT. Indeed, UCP1 expression was also strongly upregulated in the subcutaneous inguinal depot of mice after 3 weeks of wheel running.

From a physiological point of view, the induction of brown-like fat in response to exercise appears to be surprising due to several factors. First, exercise itself is an energy-consuming process and skeletal muscle is supplied with energy sources from other organs, such as free fatty acids released from WAT during physical activity. In this context, Kelly (2012) raised the question as to why physical activity would induce a program that burns fat stores, which are needed for the exercising muscle. Secondly, it has been discussed by Cannon \& Nedergaard (2004) that there should be a diminished demand for non-shivering thermogenesis during exercise, as heat is generated by skeletal muscle contraction. In accordance, several studies on rats have demonstrated that treadmill running has no effect on Ucp1 mRNA levels in BAT (Scarpace et al. 1994, Segawa et al. 1998, De Matteis et al. 2013) as well as on BAT mass (Segawa et al. 1998). The interscapular BAT of the Pgc1a transgenic mice used by Boström et al. (2012a) was also not altered when compared with WT mice. Furthermore, even a trend toward a decrease in Ucp1 mRNA expression in BAT has been observed in rats after 3 weeks of endurance exercise (Roca-Rivada et al. 2013). By contrast, only one study demonstrated that treadmill exercise training of mice leads to enhanced expression of certain brown adipocytespecific genes in BAT (Xu et al. 2011). Thus, exercise intervention is likely to have no effect on classical BAT in rodents. Regarding the browning of WAT, an increase in the number of mitochondria and enhanced expression of brown-specific genes in the visceral epididymal WAT of mice have been demonstrated after exercise (Xu et al. 2011). Another study carried out on rats also observed induction of UCP1 expression in the visceral but not the subcutaneous WAT after 3 weeks of training (Roca-Rivada et al. 2013). Boström et al. (2012a) demonstrated the most prominent effect of exercise on the subcutaneous WAT and the data obtained were in contrast to the two abovementioned studies.

In addition to these studies on rodents, a potential induction of WAT browning in response to exercise remains to be clarified in case of humans. A recent study performing a 12-week training intervention in humans could not detect any alterations in WAT. Thus, expression of several brown adipocyte-specific marker genes, such as UCP1 and PRDM16, were not significantly changed in subcutaneous adipose tissue after the intervention (Norheim et al. 2014). Hence, studies assessing the impact of exercise on WAT browning in rodents are conflicting, especially with regard to the WAT depot that undergoes remodeling in response to exercise. Furthermore, the browning of WAT in response to exercise has not yet been demonstrated in humans and, hence, further studies in humans are necessary.

Published by Bioscientifica Ltd 


\section{Exercise regulation of FNDC5 mRNA in skeletal muscle: in vivo studies}

In the initial report by Boström et al. (2012a), irisin is proposed to be a novel PGC1 $\alpha$-dependent and exerciseresponsive myokine. This conclusion was based on the finding that skeletal muscle Fndc 5 mRNA levels from mice after 3 weeks of free wheel running were enhanced compared with sedentary mice (about 2.8-fold). Moreover, enhanced $F$ ndc 5 expression was accompanied by increased Pgc1a mRNA levels (about 2.5-fold). Additionally, the expression levels of FNDC5 and PGC1A were examined in muscle biopsies from human subjects before and after 10 weeks of endurance training. Expression of both genes was enhanced after the training session (about twofold) (Boström et al. 2012a).

After this initial description of FNDC5 and irisin as exercise-regulated proteins in mice and humans, FNDC5 mRNA expression in skeletal muscle was analyzed in several human exercise cohorts (Table 1). Intriguingly, most of these studies performed in humans failed to reproduce enhanced FNDC5 mRNA levels after exercise. The upregulation of FNDC5 in skeletal muscle by exercise was only demonstrated in four out of 15 studies, including the study by Boström et al. In this context, they reported the most prominent increase in FNDC 5 mRNA expression after exercise (twofold) (Boström et al. 2012a), whereas three other studies reported only a moderate increase of 1.3- to 1.4-fold (Timmons et al. 2012, Pekkala et al. 2013, Norheim et al. 2014).

Timmons et al. (2012) were the first unable to reproduce a substantial training-induced increase in FNDC5 expression, neither in young men after endurance training nor in a different cohort (20-80 years old subjects) after resistance training. However, it is possible that FNDC 5 mRNA expression can be induced by exercise in a subset of individuals, as older active subjects had a 30\% higher FNDC5 expression than sedentary control subjects, while no difference was observed in younger subjects (Timmons et al. 2012). As FNDC5 has been described to be PGC1 $\alpha$-dependent (Boström et al. 2012a), this early study by Timmons et al. (2012) has been criticized for including exercise intervention studies without reported induction of PGC1A expression in muscle (Boström et al. 2012b). Thus, a lack of PGC1 $\alpha$ induction in some of the exercise cohorts may explain the conflicting results.

In line with this suggestion, exercise intervention studies reporting increased muscle PGC1A expression would potentially clarify the relation between FNDC5 and PGC1A mRNA expression in skeletal muscle.
Recently, Norheim et al. (2014) have compared the effects of acute and chronic exercises and assessed the correlation between PGC1A and FNDC5 expression in these exercise types. Therefore, a cohort of 13 sedentary men, aged 40-65 years, underwent a 12-week intervention of combined endurance and strength training. Muscle biopsies were taken after an acute endurance workload, both before (acute) and after (chronic) the 12-week intervention period. Interestingly, after 12 weeks of exercise, PGC1 $\alpha$ was slightly activated (1.2-fold) and FNDC5 mRNA levels were additionally increased (1.4-fold) (Norheim et al. 2014), being in line with the initial report by Boström et al. However, acute exercise intervention performed by the same subjects gave different results. Regardless of the prominent increase in PGC1A mRNA levels (7.4-fold), FNDC5 mRNA expression levels did not differ at all (Norheim et al. 2014). Prominent effects on PGC1A mRNA expression have also been described in other acute exercise cohorts (twofold and fourfold (Pekkala et al. 2013)), whereas chronic exercise had a moderate effect (1.2-fold (Norheim et al. 2014) and 1.5-fold (Boström et al. 2012a)).

In conclusion, the regulation of FNDC5 expression by exercise could not be reproduced by the majority of studies performed in humans (Table 1). Furthermore, even a strong increase in gene expression of the transcriptional coactivator $P G C 1 A$ occurring after acute exercise does not necessarily lead to an activation of FNDC5 expression (Pekkala et al. 2013, Norheim et al. 2014).

\section{Exercise regulation of FNDC5 mRNA in skeletal muscle: in vitro models}

To further investigate the effect of PGC1 $\alpha$ on FNDC5 expression in human skeletal muscle cells, in vitro studies were performed on primary human myotubes (Besse-Patin et al. 2014, Raschke et al. 2013, Norheim et al. 2014).

Treatment of primary human myotubes with drugs mimicking the activation of exercise signaling pathways, namely caffeine, ionomycin, and forskolin, significantly increased PGC1A expression. However, this induction of PGC1A mRNA expression was not accompanied by enhanced FNDC5 expression. Indeed, FNDC5 mRNA was reduced after incubating the myotubes for $24 \mathrm{~h}$ with these exercise mimetics (Norheim et al. 2014). In line with this, a second study also demonstrated significantly decreased FNDC5 expression levels in primary human myotubes challenged with ionomycin and forskolin (Besse-Patin et al. 2014).

Published by Bioscientifica Ltd. 

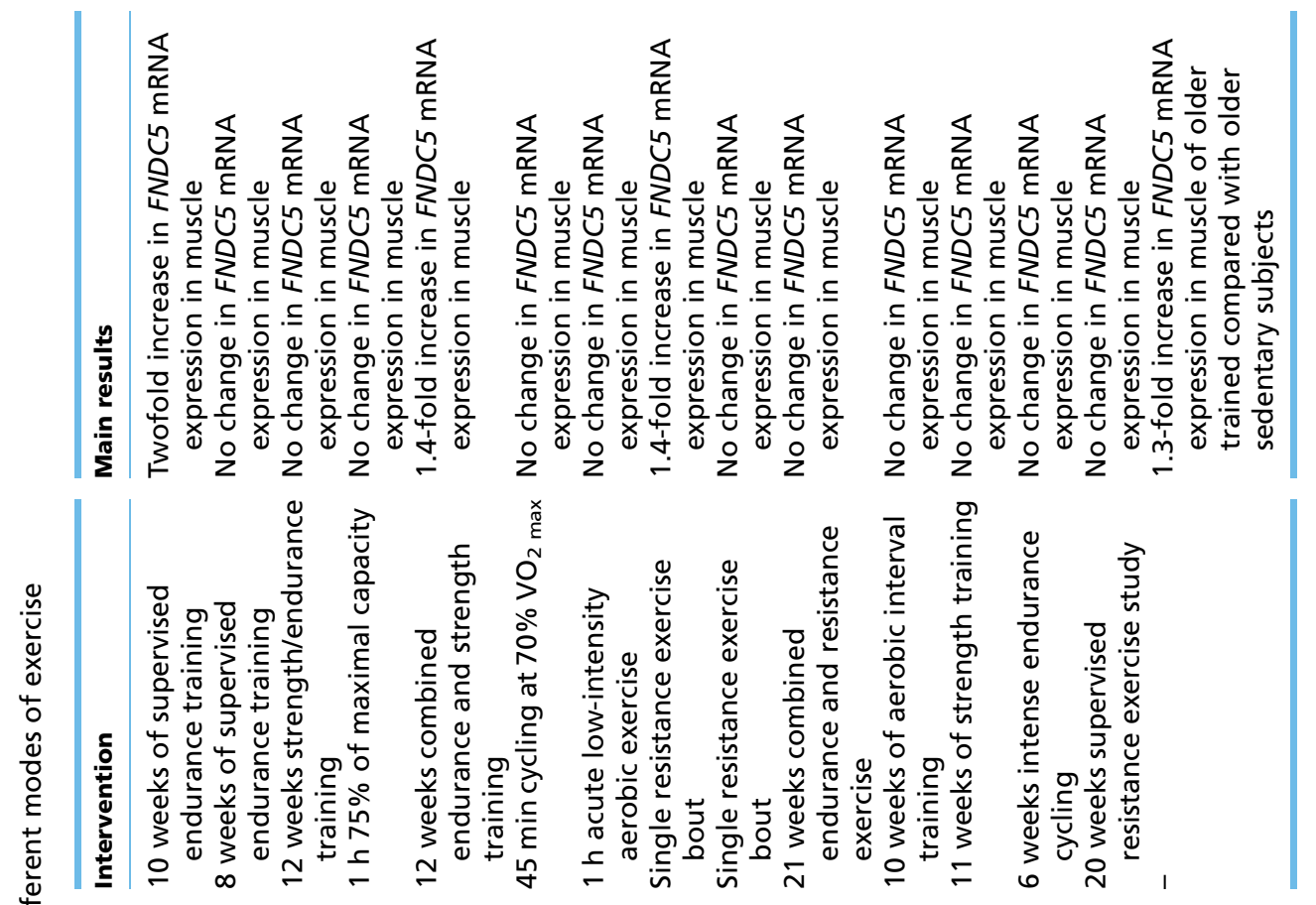

흫
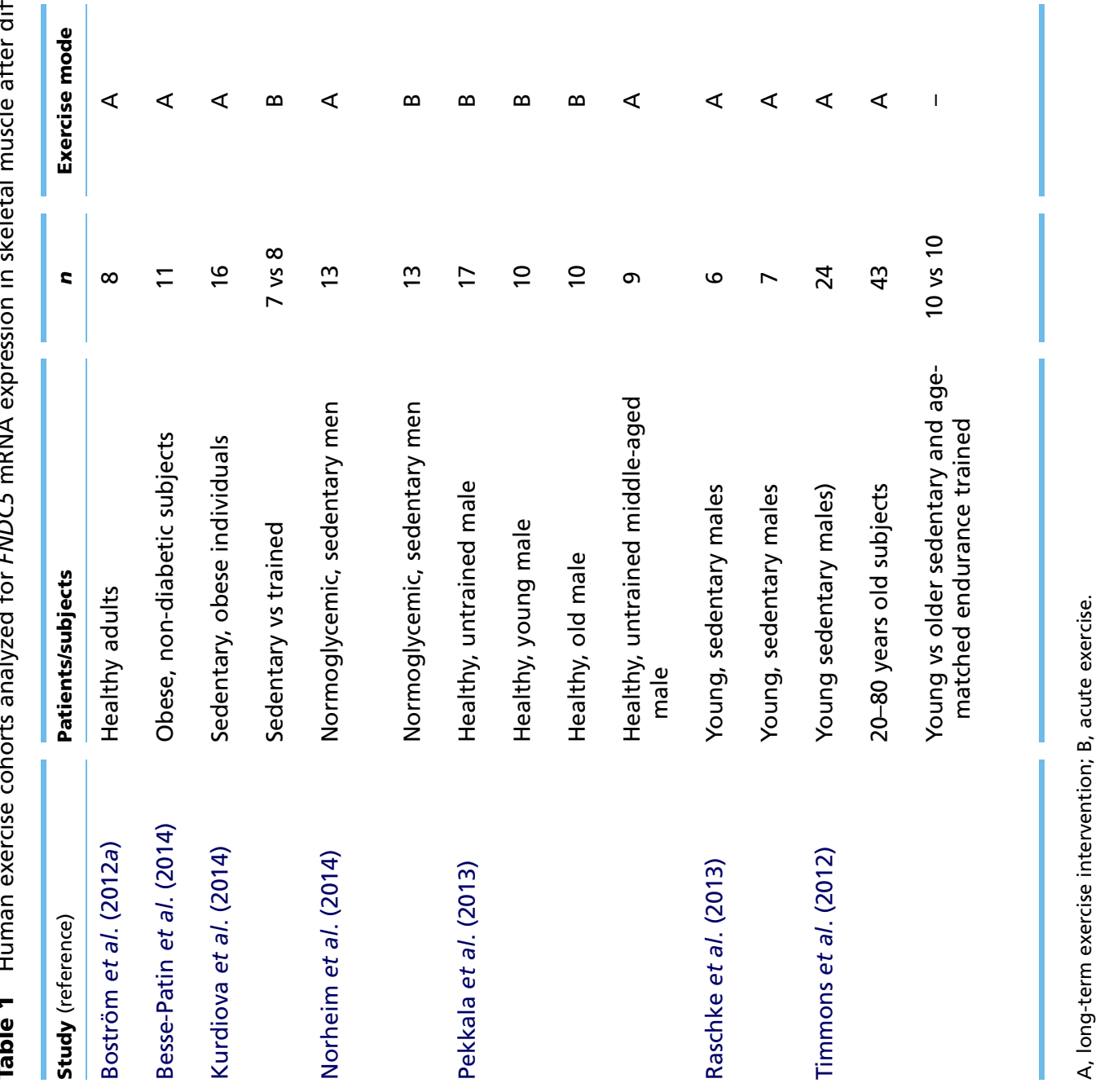
To circumvent exercise mimetics as an in vitro exercise model, electrical pulse stimulation (EPS) has been performed to induce muscle contraction in vitro. This model is well established and primary human myotubes subjected to EPS are characterized by enhanced PGC1A mRNA expression, enhanced mitochondrial biogenesis as well as enhanced secretion of the well-known myokines IL6 and VEGFA (Lambernd et al. 2012). Moreover, this EPS model reflects a training model rather than acute exercise as shown by the enhanced MYH1 mRNA level and enhanced mitochondrial content (Lambernd et al. 2012, Raschke et al. 2013). Similar to the results obtained with exercise mimetics, FNDC5 mRNA expression was not significantly enhanced in primary human myotubes after EPS, although PGC1A expression was significantly increased (Raschke et al. 2013).

To sum up, all of these in vitro studies performed with primary human skeletal muscle cells failed to demonstrate an increase in FNDC5 expression using several approaches to mimic exercise in vitro. The lack of PGC1 $\alpha$ induction as a potential reason for the absence of augmented FNDC5 expression can be excluded, as $P G C 1 A$ gene expression was significantly enhanced in all models.

Taken all described human in vivo and in vitro studies together, discussing all these discrepancies between $P G C 1 A$ activation and FNDC5 mRNA expression, Norheim et al. (2014) speculated that FNDC5 is not a direct PGC1 $\alpha$ target gene but is rather upregulated in skeletal muscle in vivo via secondary mechanisms.

The initial characterization of a candidate myokine is frequently limited to the detection of mRNA expression in skeletal muscle tissue, as it has also been done for FNDC5. Moreover, determination of gene expression or protein level in skeletal muscle biopsies is critical, as besides skeletal muscle fibers, skeletal muscle contains extended layers of connective tissues, capillaries, and nerve cells among others. Thus, gene expression studies must be followed by the detection of the encoded protein specifically in skeletal muscle fibers, e.g., by additional immunostaining of the skeletal muscle tissue sections. Finally, for full validation of a protein as a myokine, secretion from skeletal muscle cells has to be demonstrated.

\section{Exercise regulation of circulating irisin in humans}

Key points in the study by Boström et al. (2012a) were that the irisin fragment was present in the plasma of mouse and humans and that circulating levels were enhanced following exercise, as observed for skeletal muscle FNDC5 expression in this study. The presence of irisin protein in plasma was based on western blots using an antibody which detects the transmembrane segment of FNDC5 and thus would fail to detect the C-terminally cleaved, secreted irisin fragment (Erickson 2013, Raschke et al. 2013). However, later on, several ELISA assays to detect circulating irisin became commercially available and were used to quantify irisin concentrations in human exercise studies (Table 2).

Using these ELISA kits, some of these human studies reported moderately increased serum irisin levels after exercise intervention. Thus, Kraemer et al. (2014) reported transiently elevated circulating irisin levels in response to moderate aerobic exercise during the first hour after exercise (20\% increase) (Kraemer et al. 2014). In line with this finding, Huh et al. (2012) observed a moderate increase in circulating irisin level $30 \mathrm{~min}$ after a sprint running session (18\% increase) (Huh et al. 2012) and Norheim et al. (2014) demonstrated slightly increased irisin levels after $45 \mathrm{~min}$ cycling (20\% increase) (Norheim et al. 2014). In contrast to FNDC5 mRNA data, enhanced circulating irisin levels were found in acute exercise studies rather than in long-term training studies.

Despite these three reports, 12 out of 15 studies failed to demonstrate that exercise affects circulating irisin levels in humans, neither after an acute bout of exercise nor after chronic exercise training (Huh et al. 2012, Aydin et al. 2013, Hecksteden et al. 2013, Moraes et al. 2013, Pekkala et al. 2013, Kurdiova et al. 2014, Norheim et al. 2014). Recently, Hecksteden et al. (2013) have published a randomized control trial and focused on the serum irisin concentrations. Subjects performed two guidelineconforming training interventions, either endurance or strength endurance training for 26 weeks. Once again, a training-induced increase in circulating irisin levels could not be confirmed (Hecksteden et al. 2013). Interestingly, although Norheim et al. (2014) observed an increased expression of PGC1A and FNDC5 after 12 weeks of exercise, this was not translated into chronically increased levels of irisin in plasma and training for 12 weeks even tended to reduce irisin levels.

Summarizing the above-mentioned ELISA results, the current data provide equivocal results for regulation of circulating irisin levels after exercise. Only some studies reported slightly increased irisin levels after acute exercise, while the majority of studies failed to reproduce the results obtained by Boström et al. (summarized in Table 2).

Published by Bioscientifica Ltd 
Table 2 Human exercise cohorts analyzed for circulating irisin levels after different modes of exercise

\begin{tabular}{|c|c|c|c|c|c|}
\hline Study (reference) & Patients/subjects & $n$ & Exercise mode & Intervention & Main result \\
\hline Boström et al. (2012a) & Healthy adults & 8 & $A$ & $\begin{array}{l}10 \text { weeks of supervised } \\
\text { endurance training }\end{array}$ & $\begin{array}{l}\text { WB with Abnova antibody - } \\
\text { does not detect irisin }\end{array}$ \\
\hline Hecksteden et al. (2013) & $\begin{array}{l}\text { Healthy adults: } \\
\text { Control } \\
\text { Aerobic training } \\
\text { Strength training }\end{array}$ & $\begin{array}{l}39 \\
23 \\
40\end{array}$ & A & $\begin{array}{l}26 \text { weeks of supervised } \\
\text { aerobic endurance or } \\
\text { strength endurance } \\
\text { training }\end{array}$ & $\begin{array}{l}\text { No change in circulating irisin } \\
\text { levels between groups }\end{array}$ \\
\hline \multirow[t]{2}{*}{ Huh et al. (2012) } & $\begin{array}{l}\text { Young, moderately } \\
\text { trained, healthy } \\
\text { males }\end{array}$ & 15 & B & $\begin{array}{l}1 \text { week of exercise } \\
\text { ( } 2-3 \text { sets of two } 80-\mathrm{m} \\
\text { sprints) }\end{array}$ & $\begin{array}{l}\text { Circulating irisin levels were } \\
\text { significantly induced }(18 \%) \\
30 \text { min after the exercise }\end{array}$ \\
\hline & $\begin{array}{l}\text { Young, moderately } \\
\text { trained, healthy } \\
\text { males }\end{array}$ & 15 & $A$ & $\begin{array}{l}8 \text { weeks of exercise } \\
\text { (three times a week, } \\
2-3 \text { sets of two } 80-m \\
\text { sprints) }\end{array}$ & $\begin{array}{l}\text { No change in circulating irisin } \\
\text { levels }\end{array}$ \\
\hline Kraemer et al. (2014) & Healthy, young male & 7 & B & $\begin{array}{l}90 \text { min treadmill } \\
\text { exercise }\end{array}$ & $\begin{array}{l}\text { Circulating irisin levels were } \\
\text { significantly induced }(20 \%) \\
\text { by } 54 \text { min exercise }\end{array}$ \\
\hline Kurdiova et al. (2014) & Sedentary vs trained & 7 vs 8 & B & $\begin{array}{l}1 \mathrm{~h} 75 \% \text { of maximal } \\
\text { capacity }\end{array}$ & $\begin{array}{l}\text { No change in circulating irisin } \\
\text { levels }\end{array}$ \\
\hline Moraes et al. (2013) & Hemodialysis patients & 26 & $A$ & $\begin{array}{l}6 \text { months supervised } \\
\text { resistance } \\
\text { exercise }\end{array}$ & $\begin{array}{l}\text { No change in circulating irisin } \\
\text { levels }\end{array}$ \\
\hline \multirow[t]{2}{*}{ Norheim et al. (2014) } & $\begin{array}{l}\text { Normoglycemic, } \\
\text { sedentary men }\end{array}$ & 13 & $A$ & $\begin{array}{l}12 \text { weeks combined } \\
\text { endurance and } \\
\text { strength training }\end{array}$ & $\begin{array}{l}\text { No change in circulating irisin } \\
\text { levels }\end{array}$ \\
\hline & $\begin{array}{l}\text { Normoglycemic, } \\
\text { sedentary men }\end{array}$ & 13 & B & $\begin{array}{l}45 \text { min cycling at } 70 \% \\
\mathrm{VO}_{2 \max }\end{array}$ & $\begin{array}{l}\text { 1.2-fold increase in circulating } \\
\text { irisin levels directly after } \\
\text { exercise }\end{array}$ \\
\hline \multirow[t]{5}{*}{ Pekkala et al. (2013) } & $\begin{array}{l}\text { Healthy, untrained } \\
\text { male }\end{array}$ & 17 & B & $\begin{array}{l}1 \mathrm{~h} \text { acute low-intensity } \\
\text { aerobic exercise }\end{array}$ & $\begin{array}{l}\text { No change in circulating irisin } \\
\text { levels }\end{array}$ \\
\hline & Healthy, young male & 14 & B & $\begin{array}{l}\text { Single resistance } \\
\text { exercise bout }\end{array}$ & $\begin{array}{l}\text { No change in circulating irisin } \\
\text { levels }\end{array}$ \\
\hline & Healthy, young male & 10 & B & $\begin{array}{l}\text { Single resistance } \\
\text { exercise bout }\end{array}$ & $\begin{array}{l}\text { No change in circulating irisin } \\
\text { levels }\end{array}$ \\
\hline & $\begin{array}{l}\text { Healthy, untrained } \\
\text { middle-aged male }\end{array}$ & 9 & $A$ & $\begin{array}{l}21 \text { weeks high-intensity } \\
\text { endurance exercise }\end{array}$ & $\begin{array}{l}\text { No change in circulating irisin } \\
\text { levels }\end{array}$ \\
\hline & $\begin{array}{l}\text { Healthy, untrained } \\
\text { middle-aged male }\end{array}$ & 9 & $A$ & $\begin{array}{l}21 \text { weeks combined } \\
\text { endurance and } \\
\text { resistance exercise }\end{array}$ & $\begin{array}{l}\text { No change in circulating irisin } \\
\text { levels }\end{array}$ \\
\hline Aydin et al. (2013) & $\begin{array}{l}\text { Obese males vs healthy } \\
\text { males }\end{array}$ & 7 vs 7 & B & $\begin{array}{l}45 \text { min of moderate } \\
\text { outdoor running } \\
(5.5 \mathrm{~km} / 45 \mathrm{~min})\end{array}$ & $\begin{array}{l}\text { No change in circulating irisin } \\
\text { levels }\end{array}$ \\
\hline
\end{tabular}

A, long-term exercise intervention; B, acute exercise.

\section{Impact of irisin on WAT browning}

The idea of irisin as an exercise-regulated myokine in humans and the physiological role of exercise-mediated browning of WAT have been discussed in the previous section. Independent of the potential regulation of circulating irisin and skeletal muscle FNDC5 expression in response to acute or chronic exercise, the functionality of irisin in humans remains to be elucidated. Boström et al. (2012a) initially demonstrated that recombinant irisin and FNDC5 induce browning of WAT-derived murine preadipocytes in vitro. In the context of irisin as a therapeutic approach in the fight against obesity and its associated metabolic diseases, it is crucial to prove that irisin has an effect on white-to-brown transition in human cell models. Therefore, functional studies investigating the potency of irisin as an inducer of browning, performed in rodents and humans, will be discussed in this section.

After selection of secreted PGC1 $\alpha$ - and exerciseregulated proteins/myokines in mice, Boström et al. (2012a) assessed their potential as inducers of browning and identified FNDC5 as a promising candidate. To study browning of WAT in vitro, murine preadipocytes were isolated from the inguinal fat depot and treated with commercially available recombinant FNDC5 (Abnova, 
Taiwan, China) during adipogenic differentiation. The inguinal depot is regarded as a subcutaneous and generally white fat depot, but has a high ability to undergo browning in response to a cold environment (Walden et al. 2012) and hormonal stimuli such as BMP7 (Schulz et al. 2011). Treatment of primary murine subcutaneous preadipocytes with $20 \mathrm{nM}$ recombinant FNDC5 increased the expression of the BAT marker genes Ucp1, Cidea, and Pgc1a as well as oxygen consumption as a functional readout (Boström et al. 2012a). By contrast, FNDC5 failed to enhance brown marker genes in classical brown adipocytes isolated from the interscapular depot, suggesting tissue- and/or lineage-specific effects for FNDC5. The beneficial effect of irisin has also been demonstrated in vivo, as high-fat diet-induced obesity was reduced by adenoviral-mediated overexpression of FNDC5 in mice (Boström et al. 2012a).

The idea of a tissue-dependent impact of FNDC5 on browning was further elucidated by a second study from the same group. They proposed that brite or beige adipocytes display a distinct subpopulation within white adipose tissue and are highly responsive to $\beta$-adrenergic stimulation similar to classical brown adipocytes (Wu et al. 2012). In this context, CD137 (TNFRSF9) and TMEM26 were identified as selective cell surface marker genes highly expressed in brite adipocytes, but with a low expression in classical brown and white adipocytes. Interestingly, CD137-sorted cells from the stromal vascular fraction isolated from the inguinal depot of mice display a strong browning response toward 20 nM FNDC5 (Abnova) or $100 \mathrm{nM}$ of the fusion protein irisin-Fc. On the other hand, cells expressing CD137 to a lesser extent did not show any significant response toward FNDC5 or irisin-Fc, indicating that only a subpopulation of preadipocytes isolated from the subcutaneous depot, which highly express CD137, is responsible for the browning effect of irisin (Wu et al. 2012).

Another study using murine 3T3-L1 adipocytes and primary rat adipocytes also observed a white-to-brown shift after exposure to $20 \mathrm{nM}$ recombinant irisin, which was produced in yeast using the human irisin cDNA sequence (Zhang et al. 2014). Notably, the irisin-mediated increase in Ucp1 mRNA expression was higher in 3T3-L1 adipocytes compared with primary rat adipocytes (sevenfold vs fourfold), indicating potential differences in the action of irisin between species. Unfortunately, CD137 expression was not assessed in this study. In line with these results in murine and rat cell systems, a third study demonstrated the impact of irisin on browning of murine WAT. Treatment of primary murine preadipocytes isolated from the inguinal depot with $20 \mathrm{nM}$ of a commercially available FNDC5 peptide (Abcam, ab117436) enhanced the expression of Ucp1 and other brown marker genes (Shan et al. 2013). However, the synthetic peptide from Abcam used for this study corresponds to the C-terminal region of human FNDC5 and comprises the amino acids 149-178. This peptide is a part of the transmembrane domain and does not share any sequences with the irisin fragment, which comprises amino acids 32-143 (UniProt entry Q8NAU1). Therefore, the results observed by Shan et al. (2013) with this peptide are surprising and not related to the irisin fragment.

Summarizing the current data assessing the role of irisin in rodents, there is evidence for an impact of irisin on the browning of WAT. However, the function of irisin has not yet been validated in a human cell system and remains unclear. In addition to the previously described lineage-selective effect of FNDC5, potential differences in the molecular signature of adipose tissue depots between mice and humans have been proposed. Thus, Wu et al. (2012) observed that human adipose tissue from the supraclavicular region, and proven to be BAT positive by $\left[{ }^{18} \mathrm{~F}\right]$ FDG-PET/CT, shares the molecular signature of murine beige adipose tissue rather than murine classical BAT with a myogenic origin (Wu et al. 2012). However, three recent studies have provided evidence for the existence of classical brown adipocytes in humans, suggesting that the human neck adipose tissue depot is composed of both classical and brite adipocytes (Cypess et al. 2013, Jespersen et al. 2013, Lidell et al. 2013). In conclusion, these studies raise the question of whether results about browning obtained in murine models can be extrapolated to the human situation.

\section{Relevance of irisin as an inducer of browning in humans}

Studies investigating the functionality of FNDC5 and/or irisin in humans are rare and criticism has been raised regarding the relevance of irisin in humans. In this context, primary human preadipocytes isolated from the subcutaneous depot of different donors did not exhibit browning in response to irisin or FNDC5 (Raschke et al. 2013). The effects of three different recombinant proteins obtained from several sources (Phoenix, Abnova, and Caymen Chemicals) were compared in this study to exclude any potential differences between the suppliers. However, none of the proteins were able to induce browning, even when applied at high concentrations $(1000 \mathrm{ng} / \mathrm{ml}$ for FNDC5 and $600 \mathrm{ng} / \mathrm{ml}$ for irisin).

Published by Bioscientifica Ltd 
Moreover, preadipocytes isolated from donors highly expressing CD137 strongly induced the browning program in response to BMP7, while no response toward irisin or FNDC5 was observed (Raschke et al. 2013).

Until recently, this was the sole study assessing the direct effect of FNDC5/irisin on browning in primary human adipocytes. More recently, a second study was published investigating the potency of FNDC5 to induce browning in primary human adipocytes from different depots. Lee et al. (2014) demonstrated a strong induction of several brown marker genes in primary human adipocytes isolated from neck biopsies after FNDC5 treatment for 6 days. Moreover, FNDC5 treatment of neck adipocytes increased basal and uncoupled oxygen consumption rates and FNDC5-treated cells were able to respond to $\beta$-adrenergic stimulation, a crucial feature of brown adipocyte functionality. As the myogenic marker ZIC1 was not detectable, these neck adipocytes represent brite adipocytes rather than the classical brown ones. Interestingly, the effect of FNDC5 was only marginal or completely absent in primary adipocytes isolated from the subcutaneous and omental depots (Lee et al. 2014). In line with the study by Raschke et al. (2013), adipocytes from the subcutaneous depot do not appear to be able to undergo browning in response to FNDC5/irisin. One explanation could be the low expression of the britespecific marker genes TMEM26 and CD137 in the subcutaneous depot compared with deep neck adipose tissue depots (Cypess et al. 2013, Lee et al. 2014). However, high CD137 expression in human subcutaneous adipose tissue was not associated with a browning effect of FNDC5/irisin (Raschke et al. 2013).

The current data dealing with the effect of FNDC5/ irisin on browning in humans lead to the suggestion that the sensitivity toward FNDC5/irisin is potentially dependent on the adipocyte lineage and in consequence the adipose tissue depot and the species (Fig. 1). Thus, FNDC5/irisin does not activate classical brown adipocytes (Boström et al. 2012a) and has no effect on pure white adipocytes (Wu et al. 2012, Raschke et al. 2013, Lee et al. 2014). It is likely that only a small subpopulation of adipocytes, which are highly expressing brite-specific markers, is responsible for the irisin effect ( $\mathrm{Wu}$ et al. 2012). In this case, the relevance of irisin in humans and its potency as a pharmacological agent to treat obesity is questionable, as the major human adipose tissue depots (subcutaneous and omental) are pure white depots with a low expression of brite-specific markers and do not undergo browning upon FNDC5/irisin treatment. Probably, a subpopulation of cells present in deep neck adipose tissue from humans, which is suggested to be composed of classical brown and brite adipocytes, responds to FNDC5/irisin. However, the contribution of these cells to whole-body energy expenditure is not clearly determined. Moreover, other candidates seem to be more promising and have been shown to induce browning in human subcutaneous adipocytes, such as CNPs (Bordicchia et al. 2012) or BMP7 (Schulz et al. 2011, Elsen et al. 2014).

\section{Role of irisin in metabolic diseases}

In addition to the discovery of irisin as an exerciseregulated myokine, which induced browning of WAT, Boström et al. (2012a) demonstrated the beneficial effects of irisin on whole-body metabolism. Thus, adenoviralmediated FNDC5 overexpression led to browning of WAT in lean mice as well as diet-induced obese mice. In addition, a moderate increase in circulating irisin levels by threefold augmented energy expenditure, reduced the body weight gain under high-fat diet, and improved dietinduced insulin resistance (Boström et al. 2012a). These results suggested a potential protective role of irisin in the development of type 2 diabetes, one of the major obesityassociated metabolic diseases. In order to investigate the relevance of irisin in humans, many clinical studies have focused on the relation between circulating irisin levels and metabolic parameters and diseases. The following section will give a comprehensive overview about the correlation of circulating irisin with metabolic parameters in humans and highlight existing discrepancies (Table 3).

As irisin has initially been described to protect against diet-induced weight gain, mediated by browning of WAT and thus increased energy expenditure, many studies have investigated the correlation of circulating irisin with obesity in humans. In line with the suggested protective role of the myokine irisin in the development of obesity, negative correlations of circulating irisin levels with the BMI have been reported in humans (Aydin et al. 2013, Choi et al. 2013, Moreno-Navarrete et al. 2013, Polyzos et al. 2014). However, controversy exists regarding the relation between irisin levels and the BMI. Several studies reported a positive correlation of serum irisin levels with BMI (Liu et al. 2013, Stengel et al. 2013, Crujeiras et al. 2014a, Liu et al. 2014, Park et al. 2014) or could not detect a change in circulating irisin in obesity (Huh et al. 2012, Gouni-Berthold et al. 2013, Kurdiova et al. 2014). This could be related to different populations analyzed in the different studies, as some include obese subjects without

Published by Bioscientifica Ltd. 


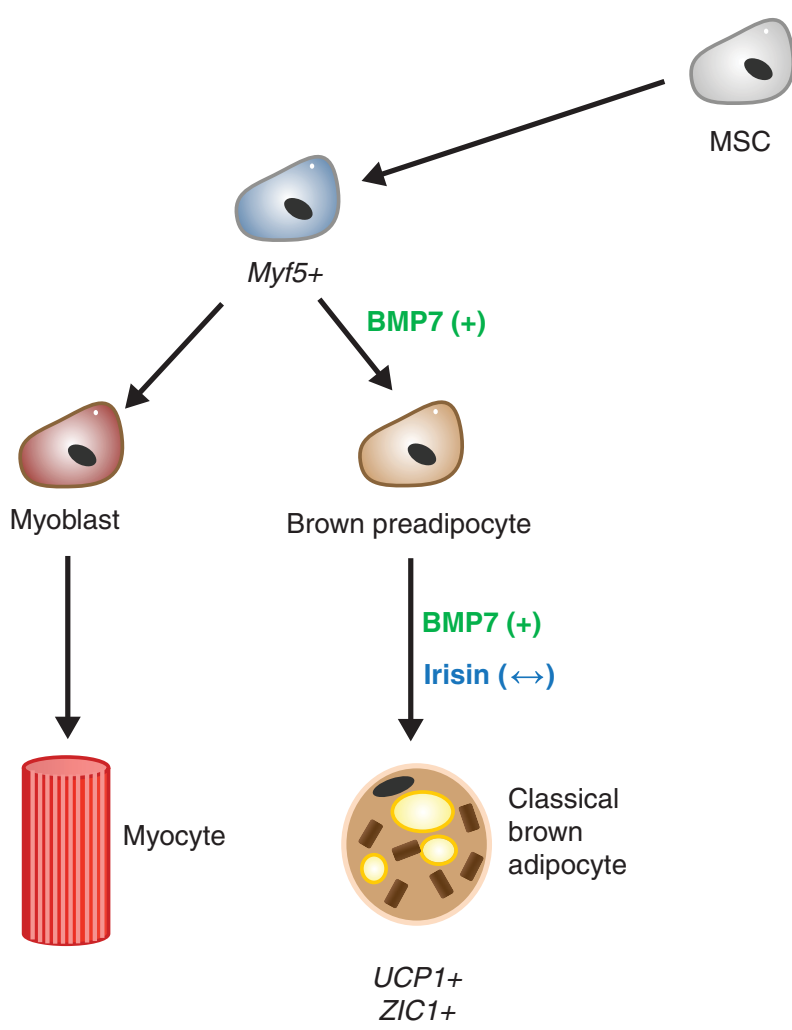

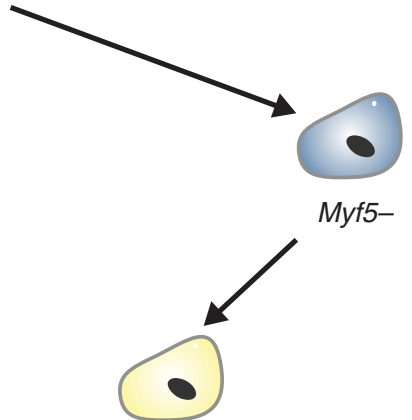
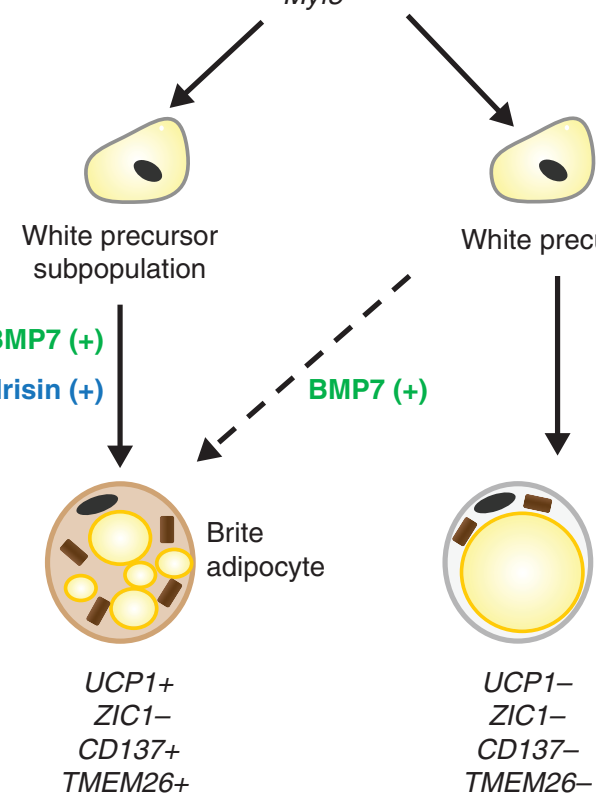

White precursor

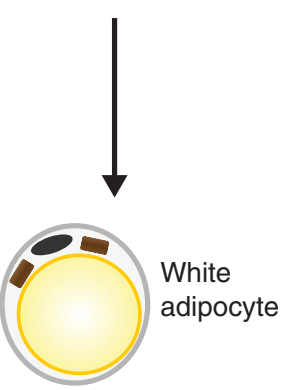

UCP1ZIC1CD137TMEM26-
Figure 1

Classical brown adipocytes share a developmental origin with skeletal muscle cells and are derived from Myf5+ precursor cells. BMP7 is involved in lineage determination as well as differentiation of classical brown adipocytes which express UCP1 and myogenic markers like ZIC1, while irisin does not promote classical brown adipocyte differentiation. White adipocytes develop from Myf5- precursor and give also rise to beige

metabolic disorders whereas others enclose obese patients with metabolic diseases such as type 2 diabetes.

However, recent intervention studies provide evidence for a positive correlation between BMI and circulating irisin levels, which is in conflict with the proposed anti-obesity effect of irisin. The earliest intervention study by Huh et al. (2012) assessed morbidly obese subjects undergoing gastric banding or gastric bypass and blood samples were collected at baseline and 6 months after surgery. Bariatric surgery led to a significant weight loss after 6 months, which was accompanied by decreased circulating irisin levels. These lower irisin levels were attributed to a lower fat-free mass and decreased FNDC5 mRNA expression in skeletal muscle (Huh et al. 2012). However, the reduction of fat mass was not analyzed as a factor causing lower circulating irisin levels. The recent observation that irisin is not only a myokine but also an adipokine (Roca-Rivada et al. 2013) raises the question if adipocytes in response to certain stimuli, such as BMP7. Only a subpopulation of white precursor cells, potentially characterized by high expression of beige markers such as CD137 and TMEM26, is likely to undergo browning in response to irisin, as proposed by Wu et al. (2012). $(+)$, positive effect; $(\leftrightarrow)$, no effect.

circulating irisin can be solely attributed to skeletal muscle. Moreover, secretion of irisin is higher from WAT of diet-induced obese rats compared with lean controls (Roca-Rivada et al. 2013), suggesting that adipose tissue, especially in states of obesity, represents an important source of irisin besides skeletal muscle. Two other interventional studies, both with 8 weeks of hypocaloric diet, observed decreased irisin levels after weight loss (de la Iglesia et al. 2013, Crujeiras et al. 2014b). In addition to a positive association between irisin and BMI, irisin has been described to be positively associated with waist circumference and fat mass. (Crujeiras et al. 2014a). In summary, there is stronger evidence for a positive correlation of circulating irisin with adiposity markers. Nevertheless, data are still conflicting and it is not clear which organ is the major source of irisin.

Besides the association of irisin with obesity, many studies assessed the regulation of irisin in type 2 diabetes

Published by Bioscientifica Ltd. 
Table 3 Correlation of circulating irisin with metabolic parameters

\begin{tabular}{l} 
Metabolic parameters \\
\hline BMI \\
T2DM \\
GDM \\
MetS \\
HOMA-IR
\end{tabular}

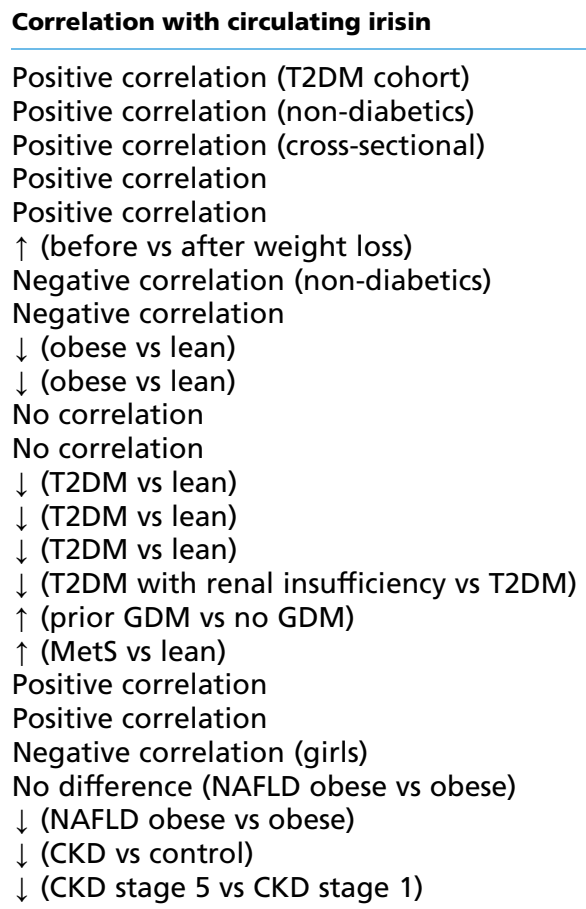

\section{References}

Liu et al. (2014)

Liu et al. (2013)

Park et al. (2014)

Stengel et al. (2013)

Crujeiras et al. (2014a)

de la Iglesia et al. (2013)

Moreno-Navarrete et al. (2013)

Choi et al. (2013)

Polyzos et al. (2014)

Aydin et al. (2013)

Huh et al. (2012)

Gouni-Berthold et al. (2013)

Kurdiova et al. (2014)

Choi et al. (2013)

Liu et al. (2013)

Liu et al. (2014)

Ebert et al. (2014b)

Park et al. (2013)

Park et al. (2013)

Ebert et al. (2014a)

Al-Daghri et al. (2013)

Polyzos et al. (2014)

Zhang et al. (2013)

Wen et al. (2013)

Ebert et al. (2014a)

T2DM, type 2 diabetes; GDM, gestational diabetes mellitus; MetS, metabolic syndrome; HOMA-IR, homeostasis model assessment-estimated insulin resistance; NAFLD, non-alcoholic fatty liver disease; CKD, chronic kidney disease.

and other metabolic diseases. Similar to the conflicting observations regarding BMI, which have been discussed above, controversy exists. While some studies reported decreased irisin levels in type 2 diabetic subjects compared with lean subjects (Choi et al. 2013, Liu et al. 2013, Kurdiova et al. 2014) and a negative correlation of HOMAIR with circulating irisin levels in girls (Al-Daghri et al. 2013), other studies demonstrated a positive correlation of circulating irisin with HOMA-IR (Park et al. 2013, Ebert et al. 2014a). In line, increased irisin levels were found in subjects with metabolic syndrome (Park et al. 2013) and higher circulating irisin concentrations have been reported in mothers with prior gestational diabetes (GDM) compared with those without prior GDM (Ebert et al. $2014 b$ ). Besides, reduced circulating irisin levels have been demonstrated in patients with chronic kidney disease (CKD) (Zhang et al. 2013) and non-alcoholic fatty liver disease (NAFLD) (Wen et al. 2013, Ebert et al. 2014a).

In addition to the different study populations, the different assays used to detect irisin may be a reason for these controversial results. Criticism regarding the reliability of commercially available irisin/FNDC5 antibodies and non-validated ELISA kits has already been raised (Erickson 2013). In this context, we summarized the cohort, the irisin concentrations measured, and the assay used in each study to gain an overview about the range of detected irisin concentrations (Supplementary Table 1, see section on supplementary data given at the end of this article). Interestingly, there is a wide range of irisin concentrations measured, from very low concentrations of $38.86 \pm 2.48 \mathrm{pg} / \mathrm{ml}$ (Choi et al. 2013) to relatively high concentrations of $2157.9 \pm 600.7 \mathrm{ng} / \mathrm{ml}$ (MorenoNavarrete et al. 2013) in lean individuals. The concentrations measured in these different studies differ strongly, with about 50000 times higher concentrations measured in the study by Moreno-Navarette et al. compared with those values originating from Choi et al. This tremendous difference between the circulating irisin concentrations indicates that at least some of the available ELISAs are unspecific and may have a high cross-reactivity to other proteins present in serum and plasma. Moreover, striking differences are observed even when using the same kit (Supplementary Table 1).

\section{Is the irisin fragment present in humans?}

Based on the huge variation in the current studies assessing circulating irisin concentrations in humans,

Published by Bioscientifica Ltd 
the physiological circulating irisin levels in humans remain unclear. Moreover, the term irisin is often incorrectly and indistinctly used with FNDC5. The secretion mechanism of irisin by extracellular cleavage of FNDC5 proposed by Boström et al. (2012a) and the presence of a $12.6 \mathrm{kDa}$ irisin fragment (theoretical molecular weight of the irisin chain amino acids 32-142 according to UniProt entry Q8NAU1) in the circulation has not been proven. As mentioned previously, for the initial description of irisin. an antibody raised against a peptide corresponding to amino acids $149-178$ and thus mainly located in the transmembrane domain was used (Erickson 2013, Raschke et al. 2013). This antibody (ab117436, Abcam) is not predicted to detect irisin and is no longer available at Abcam. Nevertheless, a band of $22 \mathrm{kDa}$ was designated to irisin, which was detected in western blot of deglycosylated cell media from HEK293 cells transfected with FNDC5 (Boström et al. 2012a). This molecular weight of $22 \mathrm{kDa}$ is more likely to reflect fulllength FNDC5 (23.7 kDa, according to Q8NAU1) than irisin (12.6 kDa, see above), which would not be in conflict with the used antibody. A later study by Roca-Rivada et al. (2013) used two different antibodies, one solely against full-length FNDC5 (Abcam, raised against amino acids 149-178) and the other also detecting the irisin fragment (Phoenix Pharmaceuticals, raised against amino acids 42-142), to discriminate between these two proteins. With both antibodies, a band of $25 \mathrm{kDa}$ was observed in conditioned media from rat skeletal muscle corresponding to full-length FNDC5. Interestingly, no additional band at the predicted lower molecular weight of irisin was observed with the anti-irisin antibody (Roca-Rivada et al. 2013), questioning the release of the initially described irisin fragment. Recently, a mass spectrometry approach has been used to clarify the identity of FNDC5-immunoreactive bands in human serum samples, which underwent deglycosylation. Several bands were observed with an anti-FNDC5 antibody from Abcam (ab131390) detecting both FNDC5 and the irisin fragment; but exclusively in a band of $24 \mathrm{kDa}$, a unique peptide located in the irisin sequence was identified by mass spectrometry, confirming that this band represents FNDC5 or fragments of FNDC5 (Lee et al. 2014). Unfortunately, this band was also designated as the irisin fragment, although the molecular weight is $24 \mathrm{kDa}$ and the band could also represent other forms of FNDC5. Summarizing these studies, it remains unclear which fragments of FNDC5 are present in the circulation of humans.

Additionally, sequence differences between species have been reported. FNDC5 has already been described in
2011 to contain a non-canonical ATG start codon in humans (Ivanov et al. 2011). In line, multi-species alignment of the FNDC5 exon 1 revealed that the canonical ATG start site is conserved in mouse, rat, gorilla, and chimp, but displays a mutation in the human sequence to ATA (Raschke et al. 2013). In vitro studies demonstrated that the translational efficiency of full-length FNDC5 is strikingly reduced in this case and in consequence also the release of FNDC5 fragments. Usage of the next in-frame ATG as an alternative start site would lead to a truncated form of FNDC5 lacking the first 76 amino acids (Raschke et al. 2013). Interestingly, the specific fragment in the FNDC5-immunoreactive band with a molecular weight of $24 \mathrm{kDa}$ identified by mass spectrometry (Lee et al. 2014) is a part of this truncated form (represented by NP_715637) described by Raschke et al. Taken together, FNDC5 fragments are present in humans, but the nature and the secretion mode have to be clarified in future studies and the presence of the initially described irisin peptide in humans is unclear.

\section{Summary and conclusion}

The discovery of irisin as an exercise-regulated myokine inducing browning of WAT has gained interest as a potential new strategy to combat obesity and its associated disorders, such as type 2 diabetes. However, there are inconsistencies regarding the relevance of irisin in humans. The regulation of FNDC5 mRNA expression by exercise and contraction could not be reproduced by a number of human studies using several exercise protocols and in vitro approaches. Furthermore, the nature of FNDC5 fragments and the presence of irisin in humans are questionable and probably contribute to conflicting data obtained with commercially available ELISA kits. Most importantly, the information regarding the concentration of circulating irisin in humans is not clear, as different studies using different kits measure irisin levels in a wide range. Data about the role of irisin in states of human obesity and metabolic diseases are conflicting and, in some cases, changes in irisin levels have been observed; they were only moderate with $10-20 \%$. Independent of the presence and regulation of FNDC5/irisin in humans, the application of recombinant irisin could still represent a therapeutic strategy to fight obesity. However, the current data obtained from human cell models reveal that FNDC5/irisin has no effect on browning of the major WAT depots in humans and is likely to selectively target a small subpopulation of adipocytes, located in classical BAT

Published by Bioscientifica Ltd. 
regions, such as the supraclavicular adipose tissue. Thus, other candidates, such as BMP7 or CNPs, seem to be more prominent candidates as inducers of browning in humans.

\section{Supplementary data}

This is linked to the online version of the paper at http://dx.doi.org/10.1530/ JOE-14-0189.

\section{Declaration of interest}

The authors declare that there is no conflict of interest that could be perceived as prejudicing the impartiality of the review reported.

\section{Funding}

This work was supported by the Ministerium für Wissenschaft und Forschung des Landes Nordrhein-Westfalen (Ministry of Science and Research of the State of North Rhine-Westphalia), the Bundesministerium für Gesundheit (Federal Ministry of Health), and the Leibniz Gemeinschaft (SAW-FBN-2013-3).

\section{References}

Al-Daghri NM, Alkharfy KM, Rahman S, Amer OE, Vinodson B, Sabico S, Milan P, Harte AL, McTernan PG, Alokail MS et al. 2013 Irisin as a predictor of glucose metabolism in children: sexually dimorphic effects. European Journal of Clinical Investigation [in press]. (doi:10.1111/ eci.12196)

Aydin S, Aydin S, Kuloglu T, Yilmaz M, Kalayci M, Sahin I \& Cicek D 2013 Alterations of irisin concentrations in saliva and serum of obese and normal-weight subjects, before and after $45 \mathrm{~min}$ of a Turkish bath or running. Peptides 50 13-18. (doi:10.1016/j.peptides.2013.09.011)

Besse-Patin A, Montastier E, Vinel C, Castan-Laurell I, Louche K, Dray C, Daviaud D, Mir L, Marques MA, Thalamas C et al. 2014 Effect of endurance training on skeletal muscle myokine expression in obese men: identification of apelin as a novel myokine. International Journal of Obesity 38 707-713. (doi:10.1038/ijo.2013.158)

Bordicchia M, Liu D, Amri EZ, Ailhaud G, Dessi-Fulgheri P, Zhang C, Takahashi N, Sarzani R \& Collins S 2012 Cardiac natriuretic peptides act via 38 MAPK to induce the brown fat thermogenic program in mouse and human adipocytes. Journal of Clinical Investigation 122 1022-1036. (doi:10.1172/JCI59701)

Boström P, Wu J, Jedrychowski MP, Korde A, Ye L, Lo JC, Rasbach KA, Bostrom EA, Choi JH, Long JZ et al. 2012a A PGC1- $\alpha$-dependent myokine that drives brown-fat-like development of white fat and thermogenesis. Nature 481 463-468. (doi:10.1038/nature10777)

Boström P, Wu J, Jedrychowski MP, Korde A, Ye L, Lo JC, Rasbach KA, Bostrom EA, Choi JH, Long JZ et al. $2012 b$ Boström et al. reply. Nature 488 E10-E11. (doi:10.1038/nature11365)

Cannon B \& Nedergaard J 2004 Brown adipose tissue: function and physiological significance. Physiological Reviews 84 277-359. (doi:10.1152/physrev.00015.2003)

Choi YK, Kim MK, Bae KH, Seo HA, Jeong JY, Lee WK, Kim JG, Lee IK \& Park KG 2013 Serum irisin levels in new-onset type 2 diabetes. Diabetes Research and Clinical Practice 100 96-101. (doi:10.1016/j.diabres.2013. 01.007)

Crujeiras AB, Pardo M, Arturo RR, Santiago NC, Zulet MA, Martinez JA \& Casanueva FF 2014a Longitudinal variation of circulating irisin after an energy restriction-induced weight loss and following weight regain in obese men and women. American Journal of Human Biology 26 198-207. (doi:10.1002/ajhb.22493)

Crujeiras AB, Zulet MA, Lopez-Legarrea P, de la Iglesia I, Pardo M, Carreira MC, Martinez JA \& Casanueva FF 2014b Association between circulating irisin levels and the promotion of insulin resistance during the weight maintenance period after a dietary weight-lowering program in obese patients. Metabolism 63 520-531. (doi:10.1016/j. metabol.2013.12.007)

Cypess AM, Lehman S, Williams G, Tal I, Rodman D, Goldfine AB, Kuo FC, Palmer EL, Tseng YH, Doria A et al. 2009 Identification and importance of brown adipose tissue in adult humans. New England Journal of Medicine 360 1509-1517. (doi:10.1056/NEJMoa0810780)

Cypess AM, White AP, Vernochet C, Schulz TJ, Xue R, Sass CA, Huang TL, Roberts-Toler C, Weiner LS, Sze C et al. 2013 Anatomical localization, gene expression profiling and functional characterization of adult human neck brown fat. Nature Medicine 19 635-639. (doi:10.1038/ $\mathrm{nm} .3112)$

De Matteis R, Lucertini F, Guescini M, Polidori E, Zeppa S, Stocchi V, Cinti S \& Cuppini R 2013 Exercise as a new physiological stimulus for brown adipose tissue activity. Nutrition, Metabolism, and Cardiovascular Diseases 23 582-590. (doi:10.1016/j.numecd.2012.01.013)

Ebert T, Focke D, Petroff D, Wurst U, Richter J, Bachmann A, Lossner U, Kralisch S, Kratzsch J, Beige J et al. 2014a Serum levels of the myokine irisin in relation to metabolic and renal function. European Journal of Endocrinology 170 501-506. (doi:10.1530/EJE-13-1053)

Ebert T, Stepan H, Schrey S, Kralisch S, Hindricks J, Hopf L, Platz M, Lossner U, Jessnitzer B, Drewlo S et al. 2014b Serum levels of irisin in gestational diabetes mellitus during pregnancy and after delivery. Cytokine 65 153-158. (doi:10.1016/j.cyto.2013.11.009)

Elsen M, Raschke S, Tennagels N, Schwahn U, Jelenik T, Roden M, Romacho T \& Eckel J 2014 BMP4 and BMP7 induce the white-to-brown transition of primary human adipose stem cells. American Journal of Physiology. Cell Physiology 306 C431-C440. (doi:10.1152/ajpcell. 00290.2013)

Erickson HP 2013 Irisin and FNDC5 in retrospect: an exercise hormone or a transmembrane receptor? Adipocytes 2 289-293. (doi:10.4161/adip. 26082)

Goto M, Terada S, Kato M, Katoh M, Yokozeki T, Tabata I \& Shimokawa T 2000 cDNA cloning and mRNA analysis of PGC-1 in epitrochlearis muscle in swimming-exercised rats. Biochemical and Biophysical Research Communications 274 350-354. (doi:10.1006/bbrc.2000.3134)

Gouni-Berthold I, Berthold HK, Huh JY, Berman R, Spenrath N, Krone W \& Mantzoros CS 2013 Effects of lipid-lowering drugs on irisin in human subjects in vivo and in human skeletal muscle cells ex vivo. PLOS ONE $\mathbf{8}$ e72858. (doi:10.1371/journal.pone.0072858)

Hecksteden A, Wegmann M, Steffen A, Kraushaar J, Morsch A, Ruppenthal S, Kaestner L \& Meyer T 2013 Irisin and exercise training in humans - results from a randomized controlled training trial. BMC Medicine 11 235. (doi:10.1186/1741-7015-11-235)

Huh JY, Panagiotou G, Mougios V, Brinkoetter M, Vamvini MT, Schneider BE \& Mantzoros CS 2012 FNDC5 and irisin in humans: I. Predictors of circulating concentrations in serum and plasma and II. mRNA expression and circulating concentrations in response to weight loss and exercise. Metabolism 61 1725-1738. (doi:10.1016/j.metabol.2012. 09.002)

de la Iglesia R, Lopez-Legarrea P, Crujeiras AB, Pardo M, Casanueva FF, Zulet MA \& Martinez JA 2013 Plasma irisin depletion under energy restriction is associated with improvements in lipid profile in metabolic syndrome patients. Clinical Endocrinology [in press]. (doi:10.1111/cen. 12383)

Ivanov IP, Firth AE, Michel AM, Atkins JF \& Baranov PV 2011 Identification of evolutionarily conserved non-AUG-initiated N-terminal extensions in human coding sequences. Nucleic Acids Research 39 4220-4234. (doi:10.1093/nar/gkr007)

Jespersen NZ, Larsen TJ, Peijs L, Daugaard S, Homoe P, Loft A, de Jong JJ, Mathur N, Cannon B, Nedergaard J et al. 2013 A classical brown adipose 
tissue mRNA signature partly overlaps with brite in the supraclavicular region of adult humans. Cell Metabolism 17 798-805. (doi:10.1016/j. cmet.2013.04.011)

Kelly DP 2012 Irisin, light my fire. Science 336 42-43. (doi:10.1126/science. 1221688)

Kraemer RR, Shockett P, Webb ND, Shah U \& Castracane VD 2014 A transient elevated irisin blood concentration in response to prolonged, moderate aerobic exercise in young men and women. Hormone and Metabolic Research 46 150-154. (doi:10.1055/s-0033-1355381)

Kurdiova T, Balaz M, Vician M, Maderova D, Vlcek M, Valkovic L, Srbecky M, Imrich R, Kyselovicova O, Belan V et al. 2014 Effects of obesity, diabetes and exercise on Fndc5 gene expression and irisin release in human skeletal muscle and adipose tissue: in vivo and in vitro studies. Journal of Physiology 592 1091-1107. (doi:10.1113/jphysiol. 2013.264655)

Lambernd S, Taube A, Schober A, Platzbecker B, Gorgens SW, Schlich R, Jeruschke K, Weiss J, Eckardt K \& Eckel J 2012 Contractile activity of human skeletal muscle cells prevents insulin resistance by inhibiting pro-inflammatory signalling pathways. Diabetologia 55 1128-1139. (doi:10.1007/s00125-012-2454-z)

Lee P, Linderman JD, Smith S, Brychta RJ, Wang J, Idelson C, Perron RM, Werner CD, Phan GQ, Kammula US et al. 2014 Irisin and FGF21 are cold-induced endocrine activators of brown fat function in humans. Cell Metabolism 19 302-309. (doi:10.1016/j.cmet.2013.12.017)

Lidell ME, Betz MJ, Dahlqvist LO, Heglind M, Elander L, Slawik M, Mussack T, Nilsson D, Romu T, Nuutila P et al. 2013 Evidence for two types of brown adipose tissue in humans. Nature Medicine 19 631-634. (doi:10.1038/nm.3017)

Liu JJ, Wong MD, Toy WC, Tan CS, Liu S, Ng XW, Tavintharan S, Sum CF \& Lim SC 2013 Lower circulating irisin is associated with type 2 diabetes mellitus. Journal of Diabetes and its Complications 27 365-369. (doi:10.1016/j.jdiacomp.2013.03.002)

Liu JJ, Liu S, Wong MD, Tan CS, Tavintharan S, Sum CF \& Lim SC 2014 Relationship between circulating irisin, renal function and body composition in type 2 diabetes. Journal of Diabetes and its Complications 28 208-213. (doi:10.1016/j.jdiacomp.2013.09.011)

van Marken Lichtenbelt WD, Vanhommerig JW, Smulders NM, Drossaerts JM, Kemerink GJ, Bouvy ND, Schrauwen P \& Teule GJ 2009 Cold-activated brown adipose tissue in healthy men. New England Journal of Medicine 360 1500-1508. (doi:10.1056/NEJMoa0808718)

Moraes C, Leal VO, Marinho SM, Barroso SG, Rocha GS, Boaventura GT \& Mafra D 2013 Resistance exercise training does not affect plasma irisin levels of hemodialysis patients. Hormone and Metabolic Research 45 900-904. (doi:10.1055/s-0033-1354402)

Moreno-Navarrete JM, Ortega F, Serrano M, Guerra E, Pardo G, Tinahones F, Ricart W \& Fernandez-Real JM 2013 Irisin is expressed and produced by human muscle and adipose tissue in association with obesity and insulin resistance. Journal of Clinical Endocrinology and Metabolism $\mathbf{9 8}$ E769-E778. (doi:10.1210/jc.2012-2749)

Norheim F, Langleite TM, Hjorth M, Holen T, Kielland A, Stadheim HK, Gulseth HL, Birkeland KI, Jensen J \& Drevon CA 2014 The effects of acute and chronic exercise on PGC- $1 \alpha$, irisin and browning of subcutaneous adipose tissue in humans. FEBS Letters 281 739-749. (doi:10.1111/febs.12619)

Ouellet V, Labbe SM, Blondin DP, Phoenix S, Guerin B, Haman F, Turcotte EE, Richard D \& Carpentier AC 2012 Brown adipose tissue oxidative metabolism contributes to energy expenditure during acute cold exposure in humans. Journal of Clinical Investigation 122 545-552. (doi:10.1172/JCI60433)

Park KH, Zaichenko L, Brinkoetter M, Thakkar B, Sahin-Efe A, Joung KE, Tsoukas MA, Geladari EV, Huh JY, Dincer F et al. 2013 Circulating irisin in relation to insulin resistance and the metabolic syndrome. Journal of Clinical Endocrinology and Metabolism 98 4899-4907. (doi:10.1210/jc. 2013-2373)

Park KH, Zaichenko L, Peter P, Davis CR, Crowell JA \& Mantzoros CS 2014 Diet quality is associated with circulating C-reactive protein but not irisin levels in humans. Metabolism 63 233-241. (doi:10.1016/j. metabol.2013.10.011)

Pekkala S, Wiklund PK, Hulmi JJ, Ahtiainen JP, Horttanainen M, Pollanen E, Makela KA, Kainulainen H, Hakkinen K, Nyman K et al. 2013 Are skeletal muscle FNDC5 gene expression and irisin release regulated by exercise and related to health? Journal of Physiology $5915393-5400$. (doi:10.1113/jphysiol.2013.263707)

Petrovic N, Walden TB, Shabalina IG, Timmons JA, Cannon B \& Nedergaard J 2010 Chronic peroxisome proliferator-activated receptor gamma (PPARgamma) activation of epididymally derived white adipocyte cultures reveals a population of thermogenically competent, UCP1-containing adipocytes molecularly distinct from classic brown adipocytes. Journal of Biological Chemistry 285 7153-7164. (doi:10.1074/ jbc.M109.053942)

Pilegaard H, Saltin B \& Neufer PD 2003 Exercise induces transient transcriptional activation of the PGC- $1 \alpha$ gene in human skeletal muscle. Journal of Physiology 546 851-858. (doi:10.1113/jphysiol.2002. 034850)

Polyzos SA, Kountouras J, Anastasilakis AD, Geladari EV \& Mantzoros CS 2014 Irisin in patients with nonalcoholic fatty liver disease. Metabolism 63 207-217. (doi:10.1016/j.metabol.2013.09.013)

Raschke S, Elsen M, Gassenhuber H, Sommerfeld M, Schwahn U, Brockmann B, Jung R, Wisloff U, Tjonna AE, Raastad T et al. 2013 Evidence against a beneficial effect of irisin in humans. PLOS ONE $\mathbf{8}$ e73680. (doi:10.1371/journal.pone.0073680)

Roca-Rivada A, Castelao C, Senin LL, Landrove MO, Baltar J, Belen CA, Seoane LM, Casanueva FF \& Pardo M 2013 FNDC5/irisin is not only a myokine but also an adipokine. PLOS ONE 8 e60563. (doi:10.1371/ journal.pone.0060563)

Saito M, Okamatsu-Ogura Y, Matsushita M, Watanabe K, Yoneshiro T, Nio-Kobayashi J, Iwanaga T, Miyagawa M, Kameya T, Nakada K et al. 2009 High incidence of metabolically active brown adipose tissue in healthy adult humans: effects of cold exposure and adiposity. Diabetes 58 1526-1531. (doi:10.2337/db09-0530)

Scarpace PJ, Yenice S \& Tumer N 1994 Influence of exercise training and age on uncoupling protein mRNA expression in brown adipose tissue. Pharmacology, Biochemistry, and Behavior 49 1057-1059. (doi:10.1016/ 0091-3057(94)90264-X)

Schulz TJ, Huang TL, Tran TT, Zhang H, Townsend KL, Shadrach JL, Cerletti M, McDougall LE, Giorgadze N, Tchkonia T et al. 2011 Identification of inducible brown adipocyte progenitors residing in skeletal muscle and white fat. PNAS 108 143-148. (doi:10.1073/pnas. 1010929108)

Seale P, Bjork B, Yang W, Kajimura S, Chin S, Kuang S, Scime A, Devarakonda S, Conroe HM, Erdjument-Bromage H et al. 2008 PRDM16 controls a brown fat/skeletal muscle switch. Nature 454 961-967. (doi:10.1038/nature07182)

Segawa M, Oh-Ishi S, Kizaki T, Ookawara T, Sakurai T, Izawa T, Nagasawa J, Kawada T, Fushiki T \& Ohno H 1998 Effect of running training on brown adipose tissue activity in rats: a reevaluation. Research Communications in Molecular Pathology and Pharmacology 100 77-82.

Shan T, Liang X, Bi P \& Kuang S 2013 Myostatin knockout drives browning of white adipose tissue through activating the AMPK-PGC1 $\alpha$-Fndc5 pathway in muscle. FASEB Journal 27 1981-1989. (doi:10.1096/fj.12-225755)

Stengel A, Hofmann T, Goebel-Stengel M, Elbelt U, Kobelt P \& Klapp BF 2013 Circulating levels of irisin in patients with anorexia nervosa and different stages of obesity - correlation with body mass index. Peptides 39 125-130. (doi:10.1016/j.peptides.2012.11.014)

Timmons JA, Baar K, Davidsen PK \& Atherton PJ 2012 Is irisin a human exercise gene? Nature 488 E9-10. (doi:10.1038/nature11364)

Virtanen KA, Lidell ME, Orava J, Heglind M, Westergren R, Niemi T, Taittonen M, Laine J, Savisto NJ, Enerback S et al. 2009 Functional brown adipose tissue in healthy adults. New England Journal of Medicine 360 1518-1525. (doi:10.1056/NEJMoa0808949) http://joe.endocrinology-journals.org DOI: 10.1530/JOE-14-0189 (c) 2014 Society for Endocrinology Printed in Great Britain 
Walden TB, Hansen IR, Timmons JA, Cannon B \& Nedergaard J 2012 Recruited vs. nonrecruited molecular signatures of brown, "brite", and white adipose tissues. American Journal of Physiology. Endocrinology and Metabolism 302 E19-E31. (doi:10.1152/ajpendo.00249.2011)

Wen MS, Wang CY, Lin SL \& Hung KC 2013 Decrease in irisin in patients with chronic kidney disease. PLOS ONE 8 e64025. (doi:10.1371/journal. pone.0064025)

Wu J, Bostrom P, Sparks LM, Ye L, Choi JH, Giang AH, Khandekar M, Virtanen KA, Nuutila P, Schaart G et al. 2012 Beige adipocytes are a distinct type of thermogenic fat cell in mouse and human. Cell $\mathbf{1 5 0}$ 366-376. (doi:10.1016/j.cell.2012.05.016)

Xu X, Ying Z, Cai M, Xu Z, Li Y, Jiang SY, Tzan K, Wang A, Parthasarathy S, He G et al. 2011 Exercise ameliorates high-fat diet-induced metabolic and vascular dysfunction, and increases adipocyte progenitor cell population in brown adipose tissue. American Journal of Physiology.
Regulatory, Integrative and Comparative Physiology 300 R1115-R1125. (doi:10.1152/ajpregu.00806.2010)

Zhang HJ, Zhang XF, Ma ZM, Pan LL, Chen Z, Han HW, Han CK, Zhuang XJ, Lu Y, Li XJ et al. 2013 Irisin is inversely associated with intrahepatic triglyceride contents in obese adults. Journal of Hepatology 59 557-562. (doi:10.1016/j.jhep.2013.04.030)

Zhang Y, Li R, Meng Y, Li S, Donelan W, Zhao Y, Qi L, Zhang M, Wang X, Cui T et al. 2014 Irisin stimulates browning of white adipocytes through mitogen-activated protein kinase p38 MAP kinase and ERK MAP kinase signaling. Diabetes 63 514-525. (doi:10.2337/db13-1106)

Zingaretti MC, Crosta F, Vitali A, Guerrieri M, Frontini A, Cannon B, Nedergaard J \& Cinti S 2009 The presence of UCP1 demonstrates that metabolically active adipose tissue in the neck of adult humans truly represents brown adipose tissue. FASEB Journal 23 3113-3120. (doi:10.1096/fj.09-133546)

Received in final form 24 April 2014

Accepted 28 April 2014

Accepted Preprint published online 29 April 2014
C 2014 Society for Endocrinology Printed in Great Britain
Published by Bioscientifica Ltd. 